\title{
Potential Mechanistic Profiling of an OTC Analgesic as a Cytotoxic Agent in the Treatment of Hepatocellular Carcinoma
}

\author{
Marwa E. Sayour ${ }^{*}$, Ibrahim Basheer², Rania Abd El Salam³ ${ }^{3}$ Mohamed El Yamany³, \\ Amr Badr', Ahmed Farouk Al-Sadek², Raafat El-Awady ${ }^{5}$ \\ ${ }^{1}$ National Cancer Institute, Egypt, Central Pharmacy, Cairo, Egypt. \\ ${ }^{2}$ Agricultural Research Center, Central Laboratory for Agricultural Expert Systems, Cairo, Egypt. \\ ${ }^{3}$ Faculty of Pharmacy, Cairo University, Department of Pharmacology \& Toxicology, Cairo, Egypt. \\ ${ }^{4}$ Faculty of Computers and Information, Department of Computer Science, Cairo, Egypt. \\ ${ }^{5}$ College of Pharmacy, University of Sharjah, Department of Pharmacy Practice and Pharmacotherapeutics, Sharjah, \\ United Arab Emirates.
}

\section{ABSTRACT}

While being a safe over the counter drug, paracetamol has also proved to be a cytotoxic agent for cultured hepatocellular carcinoma cells (HepG2). In order to understand the biochemical mechanisms underlying its cytotoxic ability, molecular docking of paracetamol with cyclin dependent kinase 2 protein (CDK2) and breast cancer type 2 susceptibility protein (BRCA2) plus cyclooxygenase 1 (COX1) enzyme protein was undergone. Computational simulation was performed using Schrödinger software to describe the details of binding between atoms of the active sites and paracetamol. All COX1, CDK2 and BRCA2 proteins showed binding scores with paracetamol. Their G-scores were $-5.32,-5.61$ and -6.08 respectively leading to selective inhibition of these proteins and loss of their cell cycle related activity. The binding strength of COX1 and CDK2 with paracetamol was mainly dependent on the hydrophobic residues, while that of BRCA2 was contributed to charged residues. Binding is responsible for the subsequent loss of activity of these cell cycle related proteins and eventual cancer cell death via apoptosis.

Key words: Paracetamol; Cyclooxygenase 1; Cyclin Dependent kinase 2; Breast Cancer Type 2 Susceptibility Protein; Molecular Docking

\section{INTRODUCTION}

Paracetamol, chemically named as N-(4-hydroxyphenyl)acetamide, is a widely used safe non-opioid analgesic for pain management and antipyresis as an alternative to aspirin, NSAIDs and selective COX-2 inhibitors ${ }^{1,2}$. It is considered as one of the most famous over the counter drug used for pain relief in symptomatic treatment of slight and moderate pain. Moreover, it is the drug of choice for patients in whom application of non-steroidal anti-inflammatory drugs (NSAIDs) are contraindicated ${ }^{3}$.

*Corresponding author: Marwa E. Sayour, e-mail: marwaezz@yahoo.com (Received 08 September 2017, accepted 04 December 2017) 
While being one of the oldest and most common OTC analgesics, the exact mechanism of action of paracetamol at the molecular level has not yet been elucidated ${ }^{4}$. It was found that the highly selective analgesic and antipyretic effects of paracetamol are due to its inhibitory effect on prostaglandin (PGs) synthesis via prostaglandin $\mathrm{H}$ synthase (COX) inhibition. PGs are lipid mediators derived from arachidonic acid that play central roles in the pathogenesis of inflammation, fever, pain and even cancer. PGs are generated by the oxygenation of arachidonic acid to the unstable intermediate prostaglandin $\mathrm{H} 2$ (PGH2) by prostaglandin $\mathrm{H}$ synthase (PGHS) of which there are two major isoforms; the constitutive PGSH-1 (COX 1) and the inducible PGSH-2 (COX 2).

In addition to its ability to treat pain and fever, paracetamol was found to induce apoptosis and necrosis in various types of cultured liver carcinoma cells specially HepG2 cell line ${ }^{5}$. Although considered safe at therapeutic doses, at higher doses, paracetamol produces hepatotoxic effect. The key event of paracetamol cytotoxicity is its biotransformation into the reactive metabolite $\mathrm{N}$-acetyl-parabenzoquinonimine (NAPQI) by the P450 family ${ }^{6-9}$ which can induce apoptosis or necrosis on different cell models ${ }^{10,11}$. In vivo, the balance among bioactivation, detoxification and defense/ repair mechanisms determines whether a compound will elicit a toxic effect or not. However, lack of correlation between in vivo hepatotoxicity and in vitro cytotoxicity may be a result of alteration in metabolic oxidation in vitro. Hepatoma cell lines constitute a simple, readily accessible and almost unlimited source of cells from a human liver. However, a major limitation is their reduced drug-metabolizing capability ${ }^{12}$. HepG2 cells, the most widely used for hepatotoxicity studies, showed substantially lower levels of most P450 enzymes than primary hepatocytes, which can contribute to the differences in cytotoxicity found in both cellular models ${ }^{12,13}$. Based on the two facts that paracetamol/NAPQI transfer ratio in HepG2 cells is still unveiled and the fact that drug-induced cytotoxicity can also be induced by parent drug molecule and/or its metabolite ${ }^{14}$, two-part study will deal first with the interaction of paracetamol with some intracellular cell-cycle related proteins as part one (the current study). This in turn will elucidate the biochemical mechanisms underlying the established cytotoxic action of paracetamol on HepG2 cells.

Close interplay of the different disciplines is therefore of utmost importance to tackle the problem of metabolism prediction. Events that produce hepato-cellular death following the formation of paracetamol-protein adducts are poorly understood. Understanding metabolic processes at the molecular level is of fundamental importance for successful drug discovery and development. Knowing the metabolic properties of a molecule can help to optimize the stability and consequently the in vivo halflife and risk-benefit ratio of a drug ${ }^{15}$. 
Among such proteins which are strongly related to cell cycle, CDK2 and BRCA2 were selected due to their important role in cell cycle progression and DNA repair as attractive targets for the design of anti-proliferative drugs. The current study explored whether CDK2 and BRCA2 proteins have docking sites for paracetamol which can lead to inhibition of their functions and perturbation of cancer cell cycle. The loss of their activity or function may lead to cancer cell death via apoptosis.

Computational docking has become one of the most valuable tools to understand the behavior of the binding process between intracellular proteins and a certain ligand molecule. In laboratories, it's not possible to achieve that goal via microscopic examination which can only reach final results without revealing details. Computational simulation and its applications became indispensible as they are the only tool to describe the details of the binding affinity between atoms of the active sites of target proteins and their ligands. Free-energy simulation techniques have been developed for quantitative modeling of protein-ligand interactions and the prediction of binding affinity ${ }^{16,17}$.

Molecular docking could offer more insight into understanding the protein-inhibitor interactions and structural features of protein's active site. Glide ${ }^{18-20} \mathrm{de}-$ veloped by Schrödinger, is one of the most popular docking methods. It includes preparation, docking and scoring the binding. Extra precision (XP) Glide was applied in docking to enhance sampling methods and scoring functions ${ }^{2}$.

Depending on the results of previous studies which have already proved the cytotoxic ability of paracetamol on human liver carcinoma cells ${ }^{22}$, the current study explores the possible molecular docking of paracetamol with some certain intracellular proteins. Computational docking software and algorithms were used as an in-silico experimental environment. Schrodinger's software suite ${ }^{23,24}$, including different utilities, was used to study and analyze the potential binding of paracetamol with COX1, CDK2 and BRCA2 proteins in order to recognize the mechanism underlying drug's cytotoxic effect. Deeper analysis of the resulting binding details and outcomes might be promising for future discovery of untraditional liver carcinoma treatment.

\section{METHODOLOGY}

\section{Materials}

In the current study, COX1, CDK2 and BRCA 2 were selected to undergo a dock trial with paracetamol to elucidate whether paracetamol has an inhibitory effect on them that stands for its cytotoxic action on HepG2 cells. Both CDK2 and BRCA2 were selected for this purpose due to their crucial role in cell cycle progression. In addition, COX1 was also selected for being the enzyme protein linked with paracetamol's mechanism of action. 


\section{Molecular Structures:}

A. Structures of the ligand Paracetamol (PDB ID: TYL), (Figure 1A), in addition to the three studied cellular proteins, were retrieved from the Protein Data Bank $(\mathrm{PDB})^{25}$.

B. Cyclooxygenase enzyme 1 (COX-1), the structure of prostaglandin $\mathrm{H} 2$ synthase-1 was obtained complexed with P-(2'-IODO-5'-THENOYL) hydrotropic acid (IODOSUPROFEN), (PDB ID: $\left.{ }^{P} \mathrm{PGE}\right)^{26}$. The structure is shown in Figure (1B).

C. Cyclin-Dependant Kinase 2 (CDK2), the structure of CDK2 was obtained complexed with a disubstituted 4, 6-bis anilino pyrimidine CDK4 inhibitor, (PDB ID: $1 \mathrm{HoO})^{27}$. The structure is shown in Figure $(1 \mathrm{C})$.

D. Breast Cancer type 2 susceptibility protein (BRCA2), the structure of BRCA2-DSS1 complex was obtained (PDB ID: 1 IYJ) ${ }^{28}$. The structure is shown in Figure (1D).

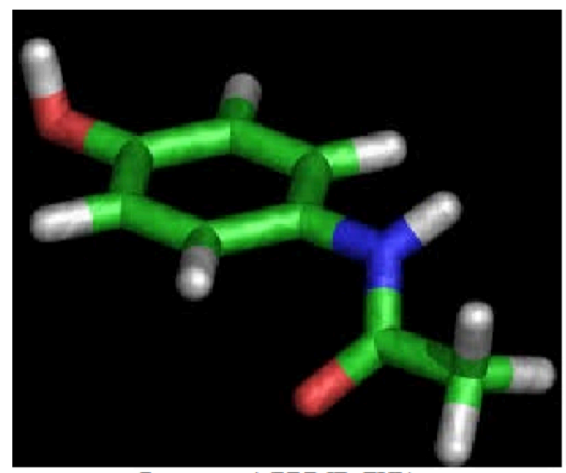

(A)Paracetamol (PDB ID: TYL)

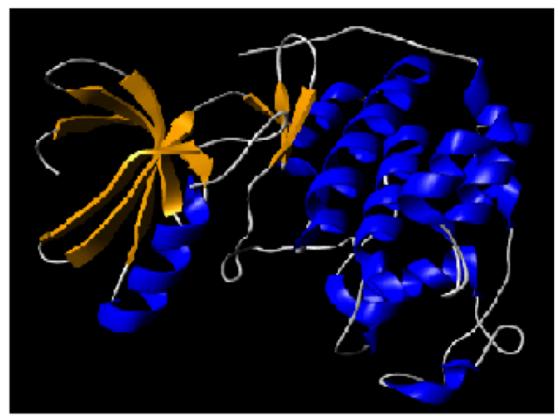

(C) CDK2 (PDB ID: 1H00)

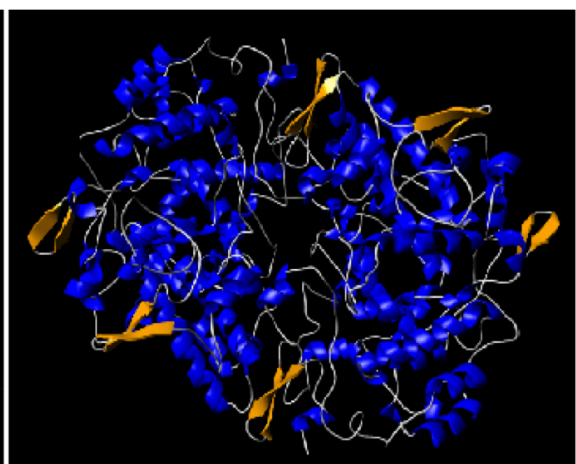

(B) COX1 (PDB ID: 1PGE)

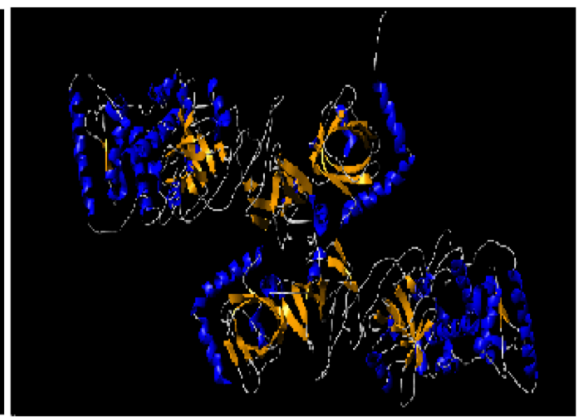

(D) BRCA2 (PDB ID:1IYJ)

Figure 1. 3D Structures of the ligand paracetamol and the 3 tested proteins 


\section{Methodology}

In the computational experiments, the methodology contains three main phases, which are preparation, binding site grid determination, and docking.

The overall flow of the docking process is shown in Figure 2. It describes the whole process and flow of steps to get the docking results as binding energy estimates.

Firstly, the preparation phase includes loading the file of the required molecule (either protein or ligand), then opening the corresponding preparation utility in Schrödinger Suite ${ }^{23,24}$, setting the required parameters and running the preparation.

Secondly, the active site of each protein is determined by running the Grid Generation module in Schrödinger Suite. Different methods were followed for each protein according to the available structure which will be explained later.

Finally, after grid generation, docking was performed with Schrödinger Glide ${ }^{18-20}$. For each protein, the ligand was docked into the prepared structure active site determined by the generated receptor grid.

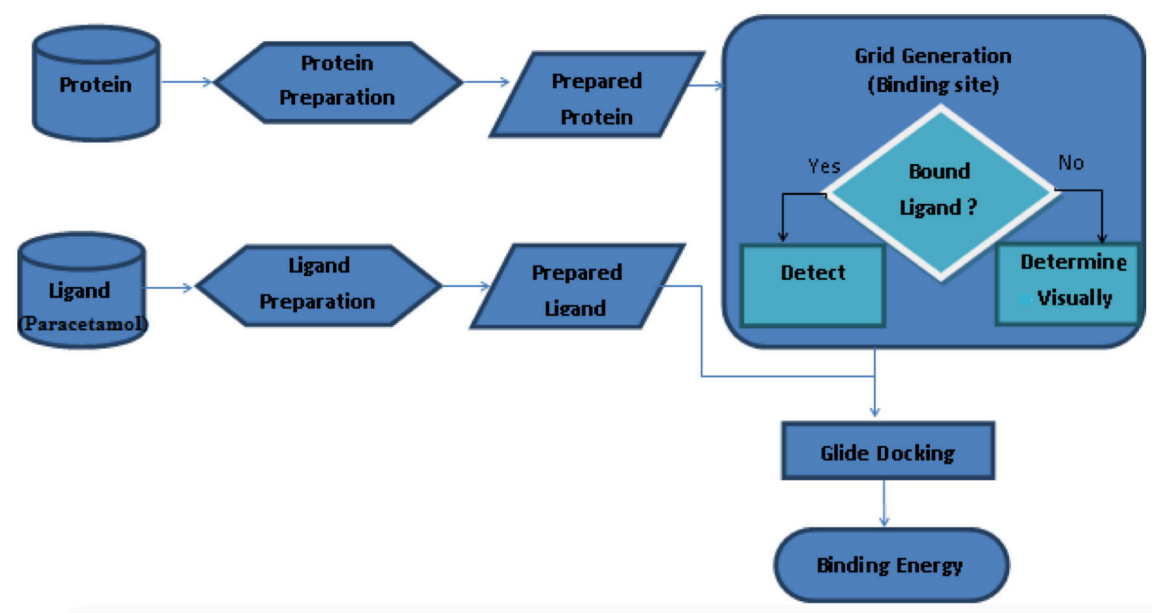

Figure 2. Docking process workflow

\section{Preparing Molecules}

The ligand, paracetamol, was loaded into Maestro 10.1 ${ }^{23,24}$, Figure 3A, and prepared by the LigPrep utility ${ }^{29}$. Force field used was OPLS_2005, and states were generated at target $\mathrm{pH}$ 7.0. The prepared structure was saved to be used in docking experiments (Figure 3B). 

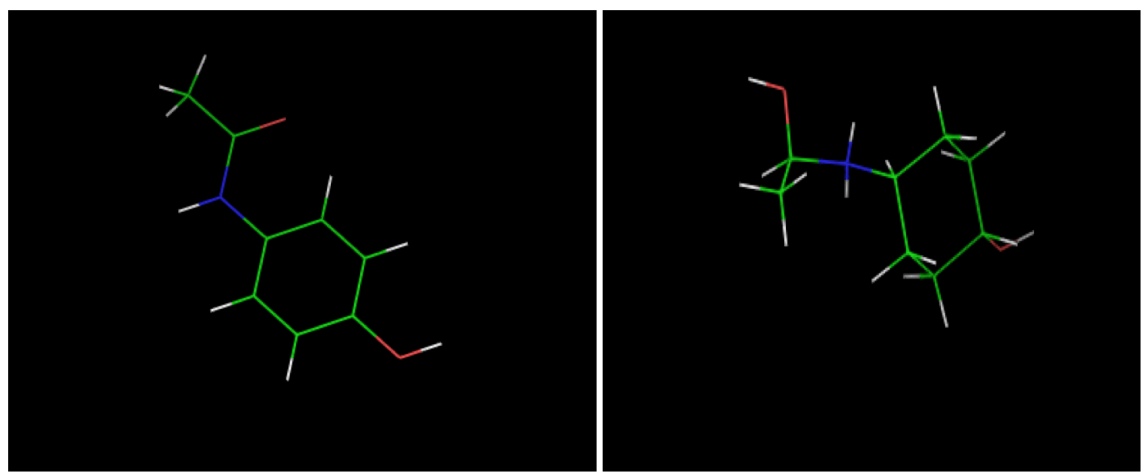

carbon, oxygen, nitrogen and hydrogen are labeled green, red, blue and white respectively

Figure 3. (A) Paracetamol before preparation (B) Paracetamol after preparation

Protein structures were prepared using the Protein Preparation utility ${ }^{30,31}$ in Schrödinger Suite. First, the structure of COX-1 protein complexed with hydrotropic acid (1PGE) was loaded into Maestro (Figure 4A). After preparation, the co-crystalized ligand [P-(2'-IODO-5'-THENOYL) hydrotropic acid] was exported as a single entry and saved in a separate file. This exported ligand was then docked back to the active site in the same way as it will be described later in the docking section. Glide uses full OPLS-AA force field at an intermediate docking stage and is claimed to be more sensitive to geometrical details compared to other docking algorithms. In the next step, water molecules were removed and $\mathrm{H}$ atoms were added to the structure, most likely positions of hydroxyl and thiol hydrogen atoms. Protonated states and tautomers of His residue and Chi 'flip' assignment for each Asn, Gln and His residue were selected by protein assignment script provided by Schrödinger. Minimization was performed until the average root mean square deviation of the nonhydrogen atoms reached $0.3 \AA^{18-21}$.

The 1 PGE structure was noticed to have two chains, A and B. Chain B and the corresponding molecules were typical as chain A. It was also observed that the bound ligands resided away from the area between the two chains. This means that the binding site is away from this area, so, the decision was taken to remove chain B for simplification, and only chain A was considered for other preparation steps for docking (Figure 4B). 

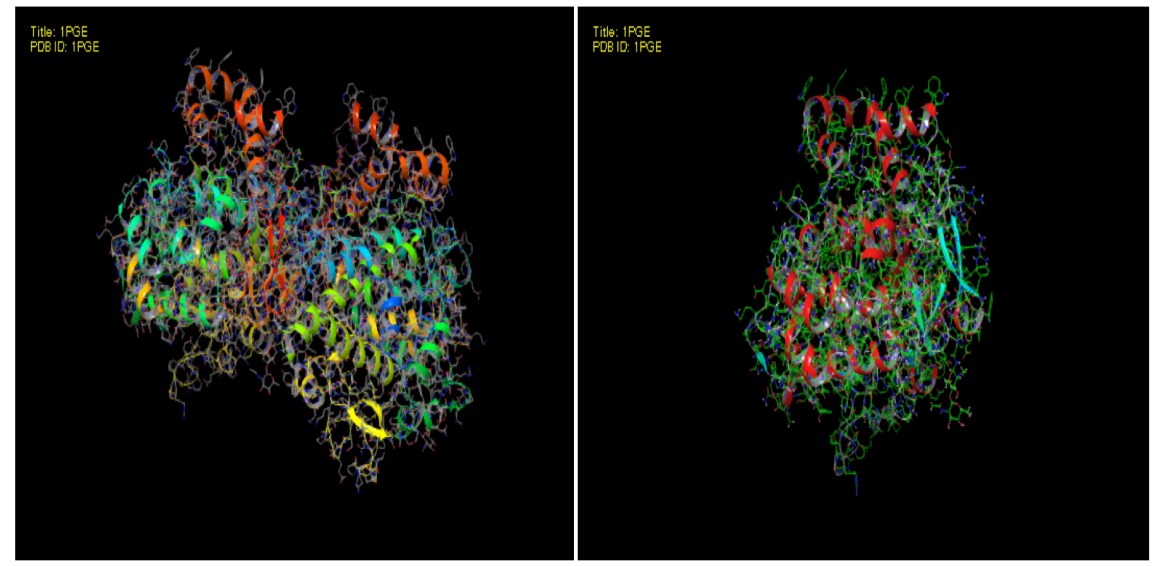

Figure 4. (A) COX1 (1PGE) before preparation (B) COX1 (1PGE) after preparation

In order to prepare the CDK2 protein, the structure of CDK2 protein complexed with a disubstituted 4, 6-bis anilino pyrimidine $\mathrm{CDK}_{4}$ inhibitor (1Hoo) was loaded. Since, this protein structure contained only one chain, no chain deletion was performed.

Regarding the BRCA2 protein, the structure of BRCA2-DSS1 protein complex (1IYJ) was loaded (Figure 5A). This protein structure contains four chains, A, B, $\mathrm{C}$, and D. Chains A and B are typical as chains $\mathrm{C}$ and D. Thus, the decision was made to delete chains $\mathrm{C}$ and $\mathrm{D}$ (Figure $5 \mathrm{~B}$ ). Additionally, as no bound ligands exist, the protein was prepared to allow for the visual determination of the binding site later.
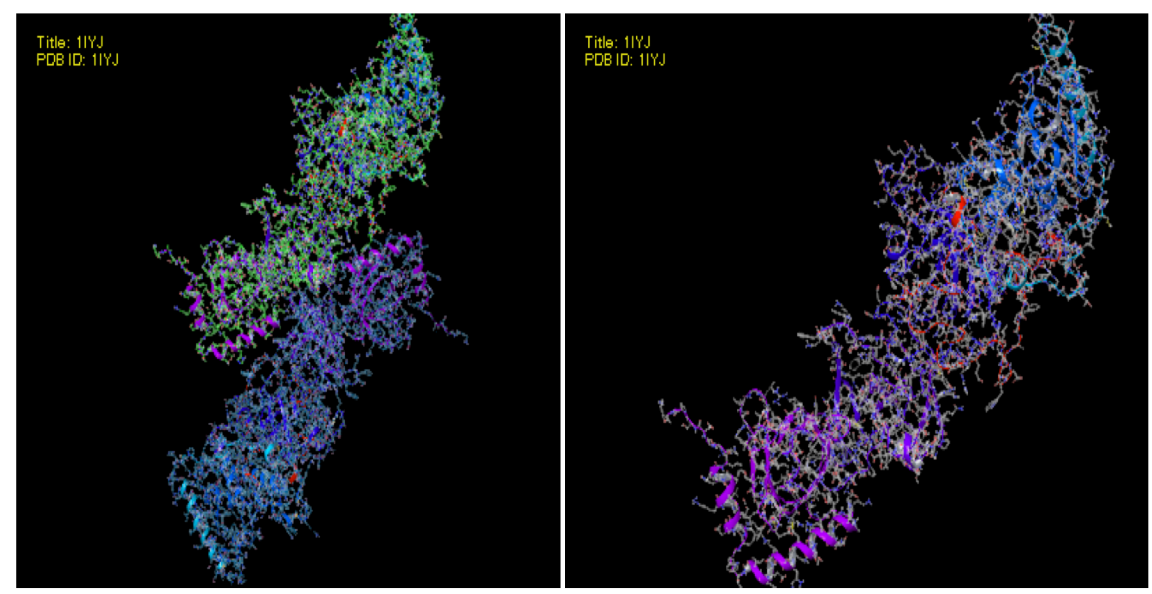

Figure 5. (A) BRCA2 (1IYJ) before preparation

(B) BRCA2 (1IYJ) after preparation 
After refining the structures and removing unnecessary chains or molecules, parameters were set and remaining steps were performed for each protein. $\mathrm{pH}$ was set to 7.0, protein structure was optimized, H-bond Assignment was applied, water removed, and Restrained Minimization was performed using OPLS_2005 force field.

\section{Grid Determination}

Receptor grid was generated for specifying the active site of the COX-1 protein. This was done using the prepared 1PGE complex structure which contains a ligand residing in the active site of the COX-1 protein. This complex structure was used to determine the active site of the protein to be used in the docking phase. The ligand was picked and the grid was determined around the picked ligand (Figure 6A).

For CDK2 protein, the receptor grid was generated for specifying the active site by using the prepared $1 \mathrm{HoO}$ complex structure, which contains a ligand residing in the active site of the CDK2 protein. The ligand was picked, and the grid was determined around the picked ligand, to be used in the docking phase (Figure 6B).

Finally, for the BRCA2 protein, the receptor grid for the active site was selected by visual inspection of the 1IYJ structure, as there was no bound ligand in the available structure. The potentially active areas of the protein were tested by setting the receptor grid manually for different times, then running the docking phase for each, and checking the results (selected grid in Figure 6C).

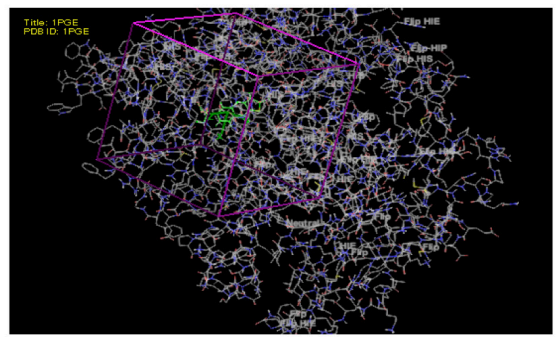

(A) $\operatorname{COX} 1$ (1PGE) Binding Grid



(B) CDK2 (1H00) Binding Grid



(C) BRCA2 (1IJY) Binding Grid

Figure 6. Binding grid for the 3 tested proteins 


\section{Docking}

The prepared proteins and the ligand obtained from Maestro 10.1, Schrödinger software was used for docking studies. The active site of the selected protein was defined to include residues within $3.5 \AA$ A radius to the complexed ligand. For docking, the authors have used Maestro scoring function with grid resolution of 0.4 $\AA$ and a flexible mode of ligand docking. The docking score was calculated as the best ligand-pose energy (kcal/mole) and the score was assessed for correlation between paracetemol and $\mathrm{COX} 1 \mathrm{CDK} 2$ and BCRA1 binding affinity and selectivity. Docking was done using Glide by loading the prepared Paracetamol file and the receptor grid file for each protein separately. For COX-1, the generated grid was loaded into glide, with paracetamol ligand. Then, the glide docking was run in the XP mode, which represents extra precession docking and more output analysis options. The XP terms and the description of their visualizations is given in Table (1). In the visualizations, hydrogen atoms are not generally displayed, and the ligand carbon atoms are colored green.

\section{The energy g score (17) is governed by the equations:}

The XP Glide scoring function is presented in equation 1. The principal terms that favor binding are presented in equation 2, while those that hinder binding are presented in equation 3 .

$\mathrm{XP}$ Glide Score $=E_{\text {coul }}+E_{\mathrm{vdW}}+E_{\text {bind }}+E_{\text {penalty------Eq-1 }}$

$E_{\text {bind }}=E_{\text {hyd_enclosure }}+E_{\text {hb_nn_motif }}+E_{\text {hb_cc_motif }}+E_{\text {pt }}+E_{\text {hb_pair }}+E_{\text {phobic_pair-Eq-2 }}$

$E_{\text {penality }}=E_{\text {desolv }}+E_{\text {ligand_strain------Eq-3 }}$

Where; G Score: Total Glide Score; sum of XP terms

Lipophilic EvdW: Lipophilic term derived from hydrophobic grid potential at the hydrophobic ligand atoms.

Phob En: Hydrophobic enclosure reward.

PhobEnHB: Reward for hydrophobically packed H-bond.

PhobEn Pair HB: Reward for hydrophobically packed correlated H-bonds.

H bond: Chem Score H-bond pair term.

Coul: Electrostatic rewards; includes Coulomb and metal terms.

Site Map: Site Map ligand-receptor non-H bonding polar-hydrophobic terms

Penaltiy: Polar atom burial and desolvation penalties, and penalty for intraligand contacts.

HB Penal: Penalty for ligands with large hydrophobic contacts and low H-bond scores. 


\section{RESULTS}

\section{Docking Analysis}

The ligand paracetamol and the crystal structure of protein complex as a template were adopted to perform the validation steps. After following the steps in the methodology section, and getting the outputs of the docking phase of this experiment, the obtained results were analyzed visually and in terms of energy values. Visual analysis helps in understanding the number of bonds, bond types, and their position. Analysis of energy values describes the strength of different energies that bind the ligand to the target protein. For visual analysis, the total glide scores of COX-1, CDK2 and BCRA2 were -5.32, -5.61 and -6.08; respectively as shown in Table (1). The total glide scores are the sum of XP terms (G Score) due to the interactions between amino acid residues of proteins with paracetamol.

The resulting redocking pose and the bonds of the co-crystalized ligand (P-(2'IODO-5'-THENOYL) hydrotropic acid) with COX-1 are the same as those used for docking paracetamol ligand as shown in (Figure $7 \mathrm{~A}, \mathrm{~B}$ ). The glide score (G score) of the co-crystalized ligand with COX-1 was -12.644 .



(A)

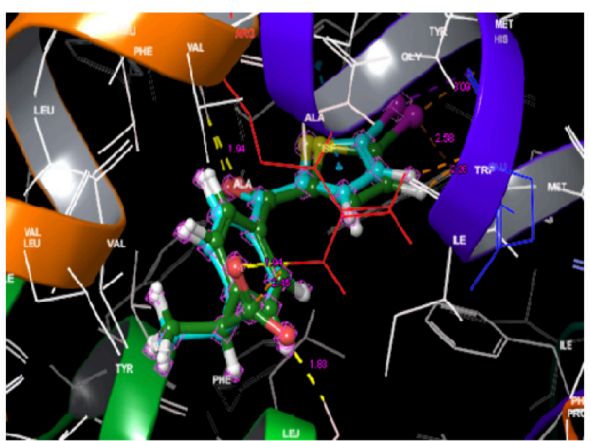

(B)

(A) Co-crystalized ligand 1PGE (labelled green)

(B) Original (labelled light blue) and redocked (labelled green) co-crystalized ligand

Figure 7. Confirmation of docking experiment 
Table 1. Comparison of binding free energies due to interaction of paracetamol with COX1, CDK2 and BCRA2.

\begin{tabular}{lccc}
\hline XP terms & COX1 & CDK2 & BCRA2 \\
\hline Total Glide Score= sum of XP terms (G Score) & -5.32 & -5.61 & -6.08 \\
\hline Dock Score & -2.78 & -5.58 & -6.05 \\
\hline Van der Waal lipophilic (EvdW) & -2.73 & -2.72 & -0.43 \\
\hline Hydrogen Bonding (HBond) & -1.79 & -1.3 & -2.94 \\
\hline Electrostatic reward (Electro) & -0.53 & -0.36 & -3.03 \\
\hline Polar interactions at the active site (Site map) & -0.29 & -0.24 & 0 \\
\hline Expos penal & 0 & 0 & 0.32 \\
\hline
\end{tabular}

The acetyl oxygen (ketonic form) of paracetamol moiety showed $\mathrm{H}$ bond interaction (distance=2.03 $\AA$ ) with the hydroxyl hydrogen of SER- COX1 protein amino residue. The phenolic oxygen of paracetomol moiety showed $\mathrm{H}$-bond interaction (distance=2.03 $\AA$ ) with the indolic hydrogen of TRP - COX1 protein amino residue. Moreover, the carboxylic oxygen (ketonic form) of LUE amino acid of the COX1 moiety showed $\mathrm{H}$ bond interaction (distance=2.35 $\AA$ ) with the phenolic hydrogen of paracetamol moiety (Figure $8 \mathrm{~A}, \mathrm{~B}$ ). The free energies of lipophilic term (LipophilicEvdW) score, ChemScore for H-bond pair term (HBond), electrostatic rewards and ligand/receptor non-Hydrogen bonding polar/hydrophobic and hydrophobic/hydrophilic complementarity terms (SiteMap) were found to be $-2.73,-1.79,-0.53$ and -0.29 respectively as seen in Table (1).

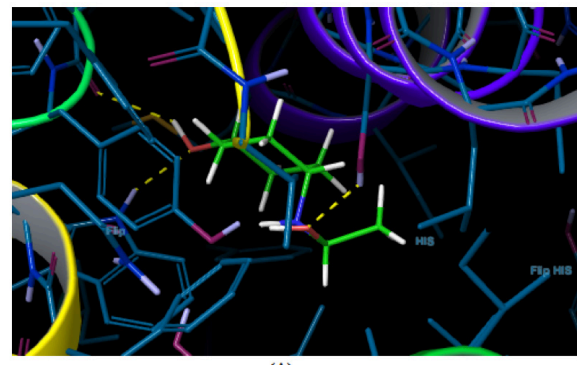

(A)

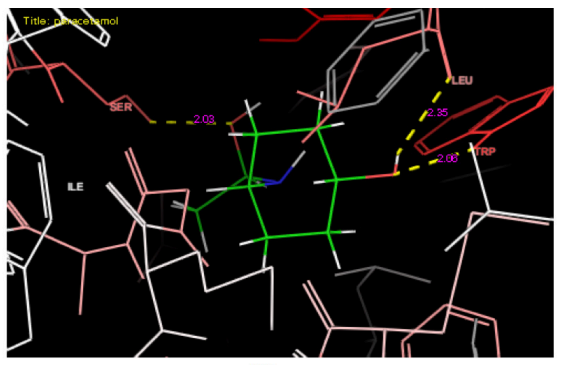

(B)

Figure 8. (A, B) Paracetamol Docked with COX1 (1PGE)

On the other hand, the detailed scores of the co-crystalized ligand [P-(2'-IODO-5'THENOYL) hydrotropic acid] attached to COX-1 for the following bonds; lipophilic term (LipophilicEvdW) score, ChemScore for H-bond pair term (HBond), electrostatic rewards and ligand/receptor non-hydrogen bonding polar/hydrophobic and hydrophobic/hydrophilic complementarity terms (SiteMap) were 
found to be $-5.8,-1.96,-0.72$ and -0.32 respectively. The resulting docking pose of [P-(2'-IODO-5'-THENOYL) hydrotropic acid] was compared with the original co-crystalized ligand (1PGE protein) using super position features in Maestro as seen in Figure 7 (B). The corresponding atoms in both poses of the ligand before co-crystalization and after redocking were selected in order and the calculated RMS for atom pairs was around 0.32. The objective for evaluating the redocking of the co-crystalised ligand was to indicate that the computational approach and the selected methodology proposed for docking was robust and could be used in the original ligand (paracetamol). The above result of RMS value (0.32) gives a level of confidence in the main docking experiment with paracetamol.

The acetyl hydrogen (enolic form) of paracetamol moiety showed $\mathrm{H}$ bond interaction (distance=1.91 $\AA$ ) with the carboxylic oxygen (ketonic form) of ASP - CDK2 protein amino residue. The phenolic hydrogen of paracetomol moiety showed H-bond interaction (distance=2.16 $\AA$ ) with the carboxylic oxygen (ketonic form) of Lue - CKD2 protein amino residue (Figure $8 \mathrm{~A}, \mathrm{~B}$ ). The free energies of lipophilic term (LipophilicEvdW) score, ChemScore for $\mathrm{H}$-bond pair term (HBond), electrostatic rewards and ligand/receptor non-Hydrogen bonding polar/hydrophobic and hydrophobic/hydrophilic complementarity terms (SiteMap) were found to be $-2.72,-1.3,-0.36$ and -0.24 respectively as seen in Table (1).

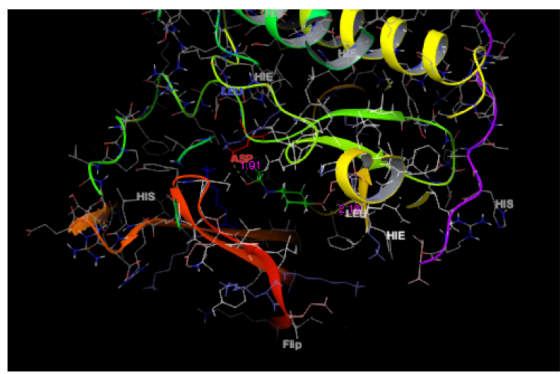

(A)

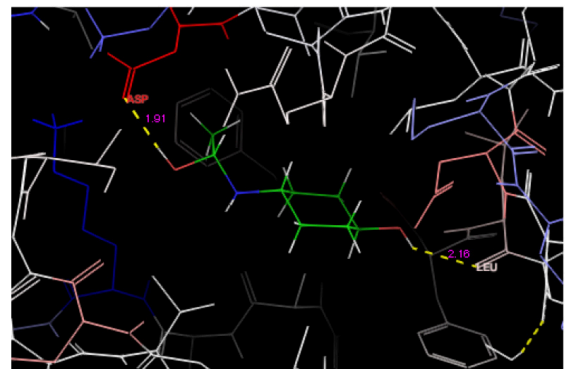

(B)

Figure 9. (A, B) Paracetamol Docked with CDK2 (1H00)

The amine hydrogens of paracetamol moiety showed $\mathrm{H}$ bond interaction (distance=1.61 and1.68 $\AA$; respectively) with the carboxylic oxygen (enolic form) of GLU- BRCA2 protein amino residue. Another H-bond interaction with the same carboxylic oxygen (enolic form) of GLU- BRCA2 protein amino residue (distance=2.47 $\AA$ ). The phenolic hydrogen of paracetomol moiety showed $\mathrm{H}$ bond interaction (distance $=1.87 \AA$ ) with the carboxylic oxygen (ketonic form) of LYS- BCRA2 protein amino residue. Finally, The phenolic oxygen of paracetamol moiety showed $\mathrm{H}$ bond interaction (distance=2.21 $\AA$ ) with the guanidine hydrogen of ARG- BCRA2 protein amino residue (Figure $9 \mathrm{~A}, \mathrm{~B}$ ). 
The free energies showed different values in case of BCRA2 interactions where lipophilic term (LipophilicEvdW) score, ChemScore for $\mathrm{H}$-bond pair term (HBond), electrostatic rewards and ligand/receptor non-hydrogen bonding polar/hydrophobic and penalty for solvent-exposed ligand groups that cancels vander Waals terms (Expos Penal) were found to be -0.43, -2.94, -3.03 and -0.24 respectively as seen in Table (1).

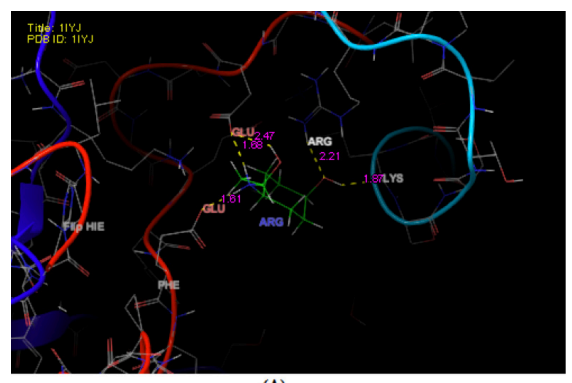

(A)

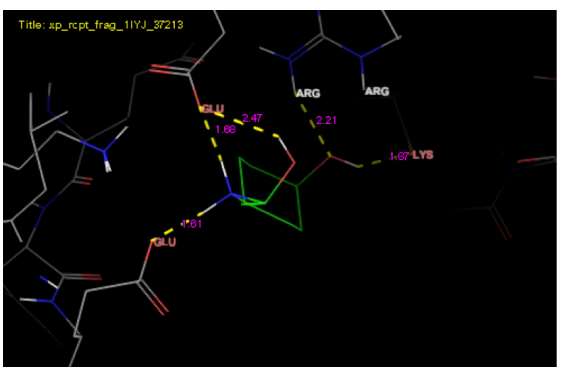

(B)

Figure 10. (A, B) Paracetamol Docked with BRCA2 (1IYJ)

The docking analysis for interactions of paracetamol and three studied proteins; COX1, CDK2 and BCRA2 are summarized in Table (2).

Table 2. Summary of overall interactions profile of paracetamol with COX1, CDK2 and BCRA2

\begin{tabular}{|c|c|c|c|c|}
\hline & Paracetamol & \multicolumn{2}{|c|}{ Amino acid residue } & Bond Length $(\AA)$ \\
\hline \multirow{3}{*}{$\operatorname{cox} 1$} & $\begin{array}{l}\text { Acetyl oxygen } \\
\text { (ketonic form) }\end{array}$ & SER & Hydroxyl hydrogen & 2.03 \\
\hline & Phenolic oxygen & TRP & Indole hydrogen & 2.06 \\
\hline & Phenolic hydrogen & LUE & ketonic carboxylic oxygen & 2.35 \\
\hline \multirow{2}{*}{ CDK2 } & $\begin{array}{l}\text { Acetyl hydrogen } \\
\text { (enolic form) }\end{array}$ & ASP & $\begin{array}{l}\text { Carboxylic oxygen } \\
\text { (ketonic form) }\end{array}$ & 1.91 \\
\hline & Phenolic hydrogen & LUE & $\begin{array}{l}\text { Carboxylic oxygen } \\
\text { (ketonic form) }\end{array}$ & 2.16 \\
\hline \multirow{5}{*}{ BCRA2 } & Amine hydrogen & \multirow{3}{*}{ GLU } & \multirow{3}{*}{$\begin{array}{l}\text { Carboxylic oxygen } \\
\text { (enolic form) }\end{array}$} & 1.61 \\
\hline & Amine hydrogen & & & 1.68 \\
\hline & $\begin{array}{l}\text { Acetyl hydrogen } \\
\text { (enol form) }\end{array}$ & & & 2.47 \\
\hline & Phenolic hydrogen & LYS & $\begin{array}{l}\text { Carboxylic oxygen } \\
\text { (ketonic form) }\end{array}$ & 1.87 \\
\hline & Phenolic oxygen & $A R G$ & Guanidine hydrogen & 2.21 \\
\hline
\end{tabular}




\section{DISCUSSION AND CONCLUSION}

As one of the common OTC analgesics, paracetamol structurally constitute an acidic moiety (anilide moiety of carboxylic acid or enols) attached to a planar, aromatic group ${ }^{32}$. The acidic group in paracetamol serves a major binding group (ionic binding) with plasma proteins. Paracetamol conjugates with proteins through amino acid linkers, where the partial charge on the donor atom binds to the hydrogen. The structure of paracetamol is N-acetyl-para-aminophenol where both acetyl and phenolic moieties segregated into two subunits opposite ends clumped together by a phenyl ring molecule in the middle.

The binding pattern of paracetamol with COX-1, CDK2 and BCRA2 proteins with their best docking pose upon comparative assessment is being carried out between the established and the novel leads ${ }^{33}$. Schrödinger software suit counted the overall binding free energy and was more efficient for analyzing the binding of equal number of van der Waals interactions formed by both proteins with the ligand molecule. The non-bonded electrostatic energy swung towards the electrostatic contribution rather than the hydrophobic one to stabilize the complexes as seen with the binding of BRCA2 with paracetamol. In general, electrostatic interactions in proteins arise due to the presence of anionic amino acids, like aspartic acid, and cationic amino acids, like lysine, arginine and glutamine. When such residues are present in close vicinity of the ligand molecule, they exert a coulombic force at a shorter distance. On examination of the interactions made by paracetamol with COX-1, CDK-2 and BCRA2, it was noticed that while the latter formed multiple electrostatic interactions, the first and the second failed to contact any positive or negative residues ${ }^{34,35}$.

Cyclin-dependent kinases (CDKs) play important roles in cell cycle progression and are attractive targets for the design of anti-proliferative drugs ${ }^{36,37}$. Many protein kinases have proved to harbor high affinity docking sites that may provide a potentially novel interface for the design of kinase inhibitors. Developing specific agents for CDK2 will assist in analyzing the role of CDK2 in cell cycle control. Moreover, BRCA2 protein is the protein product of BRCA2 gene which is responsible for DNA repairing. A Previous study reported that liver expresses BRCA2 gene which plays some role in malignancies other than breast cancer although the level of expression in the liver is much lower than in the mammary gland ${ }^{38}$.

Depending on the results of previous studies ${ }^{5,18}$ that proved the cytotoxic effect of paracetamol on HepG2 cells, the active sites of COX1, CDK2, and BRCA2 proteins were examined to figure out if there was any binding affinity between paracetamol and each of them. Results confirmed that both CDK2 and BRCA2 had 
high affinity docking sites for paracetamol which presumed them as effective targets for inhibitor design.

The proposed mechanism of paracetamol cytotoxicity might be due to the binding of paracetamol with these cell cycle related proteins which result in subsequent loss of their activity or function and eventual cell death and lysis. This comes in line with the current study as paracetamol was the fair receptor for COX1, CDK2 and BRCA2. All of them were bound to paracetmol via hydrogen bonds recording negative glide score values confirming the stability of the formed adducts.

\section{Conclusions}

Computational docking showed that the binding strength of COX1 and CDK2 with paracetamol was mainly dependent on the hydrophobic residues while that of BRCA2 was contributed to charged residues. CDK2 and BRCA2 had high affinity docking sites for paracetamol which presumed them as effective targets for inhibitor design. The binding affinity between paracetamol and the studied cell cycle-related proteins was supposed to be responsible for the subsequent loss of their activity and eventual cancer cell cycle block and death via apoptosis. In addition to our findings, further investigations are recommended to prove that docking of paracetamol with cell cycle related proteins is a promising untraditional treatment strategy for liver carcinoma in the future. Docking of the metabolite NAPQI is also recommended as a complementary study has to be done since its formation is necessary for the incidence of cytotoxicity in vivo and to some extent in vitro due to the reduced levels of P450 enzymes in HepG2 cells.

\section{ACKNOWLEDGEMENT}

The authors would like to thank Dr. Farouk's research group members, Central Laboratory for Agricultural Expert Systems, for their efforts and support during the study as a multidisciplinary team with the pharmacological team.

\section{CONFLICT OF INTEREST}

No conflict of interest is associated with this work.

\section{CONTRIBUTION OF AUTHORS}

We declare that this work was done by the authors named in this article and all liabilities pertaining to claims relating to the content of this article will be borne by them. 


\section{REFERENCES}

1. Rumack BH. Acetaminophen misconceptions. Hepatology 2004, 4O, 10-15.

2. Klotz U. Paracetamol (acetaminophen), a popular and widely used nonopioid analgesic. Arzneimittelforschung 2012, 62, 355-359.

3. Leung L. From ladder to platform: A new concept for pain management. J. Prim.Health Care 2012, 4, 254-258.

4. Howard SS. Potential analgesic Mechanisms of acetaminophen. Pain Physician 2009, 12, 269-280.

5. Manov I, Hirsh M, Iancu TC. N-acetylcysteine does not protect HepG2 cells against acetaminophen-induced apoptosis. Basic Clin Pharmacol Toxicol 2004, 94, 213-225.

6. Dai Y, Cederbaum AI. Cytotoxicity of acetaminophen in human cytochrome P4502E1-transfected HepG2 cells. J. Pharmacol. Exp. Therap. 1995, 273, 1497-1505.

7. Nelson SD. Mechanisms of the formation and disposition of reactive metabolites that can cause acute liver injure. Drug Metab.Rev. 1995, 27, 147-177.

8. Tonge RP, Kelly EJ, Bruschi SA, Kalhorn T, Eaton DL, Nebert, D, Nelson SD. Role of CYP1A2 in the hepatotoxicity of acetaminophen: investigation using Cyp1a2 null mice. Toxicol. Appl. Pharmacol 1998, 153, 102-108.

9. Manyike PT, Kharasch ED, Kalhorn TF, Slattery JT. Contribution of CYP2E1 and CYP3A to acetaminophen reactive metabolite formation. Clin. Pharmacol. Ther 2ooo, 67, 275-282.

10. Liang YL, Zhang ZH, Liu XJ, Liu XQ, Tao L, Zhang YF, Wang H, Zhang C, Chen X, Xu DX. Melatonin protects against apoptosis-inducing factor (AIF) dependent cell death during acetaminophen-induced acute liver failure. PLoSONE, 2012, 7(Pt 12), e51911.

11. Zhao X, Cong X, Zheng L, Xu L, Yin L, Peng J. Dioscin, a natural steroid saponin, shows remarkable protective effect against acetaminophen-induced liver damage in vitro and in vivo. Toxicol Lett. 2012, 214, 69-80.

12. Donato MT, Lahoz A, Castell JV, Gómez-Lechón MJ. Cell lines: a tool for in vitro drug metabolism studies. Curr Drug Metab 2008, 9, 1-11.

13. Westerink WM, Schoonen WG. Cytochrome P450 enzyme levels in HepG2 cells and cryopreserved primary human hepatocytes and their induction in HepG2 cells. Toxicol In Vitro 2007, 21, 1581-1591.

14. Gómez-Lechón MJ, Lahoz A, Gombau L, Castell JV, Donato MT. In vitro evaluation of potential hepatotoxicity induced by drugs. Current Pharmaceutical Design, 2010; Vol. 16, No.oo.

15. Kirchmair J, Göller AH, Lang D, Kunze J, Testa B, Wilson ID, Glen RC, Schneider G. Predicting drug metabolism: experiment and/or computation? Nat Rev Drug Discov. 2015, 14(6), 387-404.

16. Kollman, PA. Free energy calculations: applications to chemical and biochemical phenomena. Chem. Rev 1993, 93, 2395-2417.

17. Simonson T, Archontis G, Karplus M. Free energy simulations come of age: protein-ligand recognition. Acc. Chem. Res.2002, 35, 430-437.

18. Schrödinger Release 2015-1: Glide, Schrödinger, LLC, New York, NY, 2015.

19. Friesner RA, Banks JL, Murphy RB, Halgren TA, Klicic JJ, Mainz DT, Repasky MP, Knoll 
EH, Shaw DE, Shelley M, Perry JK, Francis P, Shenkin PS. Glide: A New Approach for Rapid, Accurate Docking and Scoring. 1. Method and Assessment of Docking Accuracy. J. Med. Chem. 2004, 47, 1739-1749.

20. Halgren TA, Murphy RB, Friesner RA, Beard HS, Frye LL, Pollard WT, Banks JL. Glide: A New Approach for Rapid, Accurate Docking and Scoring. 2. Enrichment Factors in Database Screening. J. Med. Chem. 2004, 47, 1750-1759.

21. Friesner RA, Murphy RB, Repasky MP, Frye LL, Greenwood JR, Halgren TA, Sanschagrin PC, Mainz DT. Extra Precision Glide: Docking and Scoring Incorporating a Model of Hydrophobic Enclosure for Protein-Ligand Complexes. J. Med. Chem. 20o6, 49, 6177-6196.

22. Sayour ME, Abd El Salam RM, El yamany MF, El Sayed AM, El- Awady RA..Combination of paracetamol and the glutathione depleting agent buthioninesulfoximine show differential effect on liver cancer cells and normal hepatocytes. Pharmacology\& Pharmacy 2016, 7, 443-458.

23. Schrödinger, LLC, New York, NY, 2015.

24. Schrödinger Release 2015-1: Maestro, Schrödinger, LLC, New York, NY, 2015.

25. Protein Data Bank (www.rcsb.org)

26. Loll PJ, Picot D, Garavito RM 1997. DOI: 10.2210/pdb1pge/pdb (www.rcsb.org/pdb/explore.do?structureId=1PGE).

27. Beattie JF, Breault, GA, Ellston, RPA, Green S, Jewsbury PJ, Midgley CJ, Naven RT, Minshull CA, Pauptit RA, Tucker JA, Pease JE. 2003. DOI: 10.2210/pdb1hoo/pdb (www.rcsb.org/ $\mathrm{pdb} /$ explore/explore.do?structureId=1Hoo).

28. Pavletich NP, Jeffrey PD, Yang HJ 2002. DOI: 10.2210/pdbiiyj/pdb (www.rcsb.org/pdb/ explore/explore.do?structureId=1IYJ).

29. Schrödinger Release 2015-1: LigPrep, Schrödinger, LLC, New York, NY, 2015.

30. Schrödinger Release 2015-1: Schrödinger Suite 2015-1 Protein Preparation Wizard; Epik, Schrödinger, LLC, New York, NY, 2015; Impact, Schrödinger, LLC, New York, NY, 2015; Prime, Schrödinger, LLC, New York, NY, 2015

31. Sastry GM, Day T, Adzhigirey M, Annabhimoju R, Sherman W. Protein and ligand preparation: parameters, protocols, and influence on virtual screening enrichments. J. Comput. Aided. Mol. Des. 2013, 27 (Pt 3), 221-234.

32. Asirvatham S, Dhokchawle BV, Tauro SJ. Quantitative structure activity relationships studies of non-steroidal anti-inflammatory drugs: A review. Arabian Journal of Chemistry 2016, DOI: 10.1016/j.arabjc.2016.03.002.

33. Dube PN, Mokale S, Datar P. CoMFA and docking study of 2, $\mathrm{N}^{6}$-disubstituted 1,2-dihydro1,3,5-triazine-4,6-diamines as PfDHFR enzyme inhibitors for antimalarial activity. Bulletin of Faculty of Pharmacy, Cairo University 2014, 52, 125-134.

34. Shukla S, Bafna K, Sundar D, Thorat SS. The Bitter Barricading of Prostaglandin Biosynthesis Pathway: Understanding the Molecular Mechanism of Selective Cyclooxygenase-2 Inhibition by Amarogentin, a Secoiridoid Glycoside from Swertia chirayita PLOS ONE 2014, 9 (Pt 3),1-15.

35. Agarwal S, Mishra M, Mishra VK, Kashaw V, and. Kashaw SK. Structure based molecular docking studies on some 1,3-disubstituted urea derivatives as anti-tubercular agents. IJPSR 2016, 7 (Pt 1), 228-235. 
36. Tripathi SK, Muttineni R, Singh SK. Extra precision: docking, free energy calculation and molecular dynamics simulation studies of CDK2 inhibitors. Journal of Theoretical Biology 2013, 334, 87-100

37. Ferguson M, Luciani MG, Finlan L, Ibbotson S, Fersht A, Hupp TR. The Development of a CDK2-Docking Site Peptidethat Inhibits 553 and Sensitizes Cells to Death.Cell Cycle 2oo4, 3(Pt 1), 80-89.

38. Katagiri T, Nakamura Y, Miki Y. Mutations in the BRCA2gene in hepatocellular carcinomas. Cancer Reasearch 1996, 56, 4575-4577. 